Relato de Caso

\title{
Estudo dos efeitos sensório-motores da fisioterapia aquática com pacientes em estado de coma vigil
}

\section{Study of the effect motor-sensories of the aquatic therapy with patients in vigil coma stage}

Fabio Jakaitis ${ }^{1}$, Ada Bianca Abdulmacih Guazzelli²

\begin{abstract}
RESUMO
Objetivo: Avaliar o efeito no quadro do paciente pós Traumatismo Crânio Encefálico (TCE), em estado de coma vigil, com a intervenção precoce da fisioterapia aquática. Método: $O$ estudo consiste em um relato de caso, realizado no Hospital Israelita Albert Einstein, analisando paciente pós TCE em estado de coma vigil, nível cognitivo II (Rancho Los Amigos), estabilizado clinicamente, com um ano de internação, realizando somente fisioterapia aquática. Resultado: Observou-se melhora do potencial sensorial através da conscientização espacial e do potencial motor através do controle cervical, ajuste tônico e postural e conservação das Amplitudes de Movimento (ADM's), além da redução do reflexo do membro inferior esquerdo. Conclusão: Através do presente estudo, conclui-se que o paciente em coma vigil, beneficiou-se com a intervenção da fisioterapia aquática. Devemos levar em consideração, no entanto, que em se tratando de um estudo de caso, analisamos um único paciente, desta forma não podemos nos certificar que haja benefício com um percentual significante de uma população.
\end{abstract}

Unitermos: Fisioterapia, Neurologia, Coma, Escala de Coma de Glasgow, Estimulação Física.

Citação: Jakaitis F, Guazzelli ABA. Estudo dos efeitos sensório-motores da fisioterapia aquática com pacientes em estado de coma vigil. Rev Neurociencias 2005; 13(4):215-218.

\section{SUMMARY}

Purpose: The aim of this study was to evaluate the effect in the picture of the patient after Brain Injury (BI), in vigil coma stage, with the precocious intervention of the aquatic therapy. Method: The study it consists of a story of case, carried through in the Israelita Albert Einstein Hospital, analyzing patient after BI in vigil coma stage, cognitive level II (Rancho Los Amigos), clinical stabilized, with one year of internment, only carrying through aquatic therapy. Results: Improvement of the sensories potential through the space awareness was observed and of the motor potential through the cervical control, it has adjusted to tonic and postural and conservation of the Amplitude of Movement (ADM's), beyond the reduction of the consequence of the left leg. Conclusion: Through the present study, it is concluded that the patient in vigil coma stage, was benefited with the intervention of the aquatic therapy. We must lead however in consideration, that in if treating to a case study, we analyze an only patient, of this form we cannot in certifing them that she has benefit with a significant percentage of a population.

\section{Keywords: Physical Therapy, Neurology, Coma, Glasgow Coma Scale, Physical Stimulation.}

Citation: Jakaitis F, Guazzelli ABA. Study of the effect motor-sensory of the aquatic therapy with patients in vigil coma stage. Rev Neurociencias 2005; 13(4):215-218.

Trabalho realizado: Centro de Reabilitação do Hospital Israelita Albert Einstein

1 - Fisioterapeuta do Hospital Israelita Albert Einstein, Supervisor de Estágio em Neurologia da Universidade Bandeirante de São Paulo (UNIBAN), Especialista em Fisiologia do Exercício pela Universidade Federal de São Paulo (UNIFESP)

2 - Fisioterapeuta do Hospital Israelita Albert Einstein, especialista em neurologia e fisioterapia aquática (UMESP)

Endereço para correspondência: Fabio Jakaitis

R. Dr. Roberto Feijó, 528 apto 91

Vila Prudente - São Paulo - SP - CEP: 03138-001 - Email: fjfisioterapia@ig.com.br

Trabalho recebido em 04/10/05. Aprovado em 19/12/05 
O Traumatismo Crânio Encefálico (TCE) é uma enfermidade responsável por alto índice de entrada de pacientes em prontos atendimentos, emergências e centros de reabilitação nos Estados Unidos. Representa a causa mais comum de disfunções adquiridas na infância, evoluindo com completa recuperação, importantes seqüelas físicas e cognitivas ou podendo levar até a morte ${ }^{1}$.

Nos EUA, mais de 1 milhão de crianças sofrem TCE, dentre estas 100.000 e 200.000 necessitam de hospitalização, destas, 25.000 vão a óbito e 15.000 a 30.000 sobrevivem com seqüelas físicas e/ou cognitivas, todos os anos?

No Brasil a incidência cresce a cada dia, sendo a maior causa de morte entre indivíduos na faixa etária de 10 a 29 anos. Em todo país são mais de 100.000 vítimas fatais por ano, e para cada 1 morto, 3 sobrevivem com seqüelas graves ${ }^{3}$.

O TCE tem como conceito a agressão ao cérebro não de natureza degenerativa ou congênita, mas causada por uma força física externa, que pode produzir um estado diminuído ou alterado de consciência que resulta em comprometimento das habilidades cognitivas ou do funcionamento físico, distúrbio comportamental ou emocional. Este pode ser temporário ou permanente, e provocar comprometimento funcional parcial ou total, ou mau ajuste psicológico ${ }^{4}$.

Pacientes com lesões encefálicas graves apresentam alterações sensório-motoras, negligências motoras e sensoriais, cognitivas, psicológicas, entre outras. O período prolongado no leito e a falta de estímulos sensoriais inibem e retardam o processo de evolução neuropsicomotor ${ }^{5}$.

A fisiopatologia do TCE, inclui lesões cerebrais imediatas, relacionadas ao impacto (lesão primária) e lesões tardias, relacionadas ao quadro clínico (secundária) (6). O trauma é classificado em leve, moderado ou grave $^{6}$. Os casos mais graves vêm acompanhados por períodos de coma, durando horas a longos períodos. De acordo com o tempo de manutenção neste estágio, o trauma será relacionado com a sua gravidade, segundo a Escala de Resultados de Glasgow. Esta escala serve para relacionar o paciente da fase inicial, com sua fase após 6 meses dentro de uma visão topográfica do diagnóstico ${ }^{6}$.

Dentro dos prognósticos pós-trauma, pela Escala de Glasgow, um escore 8 significa um mau prognóstico, e períodos de amnésia pós-trauma por mais de 14 dias, também indicam um mau resultado7. Para isso foi desenvolvido pelo Rancho Los Amigos, uma escala de níveis, onde se pode avaliar e classificar o paciente pós-trauma de crânio, dentro de suas funções cognitivas (tabela 1). Essa Escala foi descrita em 1965, e atualizada com mais dois itens em $2001^{8}$.
Tabela 1. Escala Rancho Los Amigos (Níveis cognitivos funcionais) ${ }^{8}$.

\begin{tabular}{|c|c|}
\hline Níveis cognitivos & Respostas funcionais \\
\hline I & Nenhuma resposta \\
\hline II & $\begin{array}{l}\text { Resposta generalizada à } \\
\text { estimulação }\end{array}$ \\
\hline III & Resposta localizada a estímulos \\
\hline IV & Comportamento confuso e agitado \\
\hline V & $\begin{array}{l}\text { Confuso, inadequado, inapropriado, } \\
\text { não agitado }\end{array}$ \\
\hline VI & $\begin{array}{l}\text { Comportamento confuso, } \\
\text { mas apropriado }\end{array}$ \\
\hline VII & $\begin{array}{l}\text { Comportamento automático e } \\
\text { apropriado }\end{array}$ \\
\hline VIII & $\begin{array}{l}\text { Comportamentos apropriados, } \\
\text { intencionais e com finalidade } \\
\text { (necessita de supervisão freqüente) }\end{array}$ \\
\hline IX & $\begin{array}{l}\text { Intencional e apropriado (supervi- } \\
\text { são quando solicitado). }\end{array}$ \\
\hline$x$ & $\begin{array}{l}\text { Intencional e apropriado } \\
\text { (independência modificada). }\end{array}$ \\
\hline
\end{tabular}

A indicação de pacientes em estágio de coma vigil (sendo este, o último estágio de inconsciência em que o paciente se encontra, antes de sair para o nível de consciência), vai depender da avaliação da equipe multidisciplinar, estabilização clínica do paciente e autorização médica e familiar ${ }^{5}$.

Após o planejamento do programa reabilitacional, o paciente começa o tratamento de acordo com suas deficiências e alterações. Dentro de cada área de atuação, seja ela médica, fisioterapêutica, aquática, terapêutica ocupacional, psicológica, fonoaudiológica, entre outras, pacientes com tempo de lesão mais recente, tem com embasamento literário, possibilidades de melhor prognóstico ${ }^{1,9-12}$.

A fisioterapia aquática, devido suas propriedades hidrostáticas, hidrodinâmicas e termodinâmicas associado aos métodos de tratamento aquático, favorecem o trabalho de estimulação sensorial, além da função. A imersão aquática possui efeitos fisiológicos relevantes que se estendem sobre todos os sistemas e a homeostase. Estes efeitos podem ser tanto imediato quanto tardio, permitindo assim fins terapêuticos no tratamento de pacientes com distúrbios músculosesqueléticos, neurológicos, cardiopulmonares, entre outros $^{13,14}$

A precocidade de início à fisioterapia aquática, após a estabilização clínica, favorece um melhor desenvolvimento neurológico, previne deformidades e a melhora do estado psicológico do paciente de seus familiares ${ }^{15}$ 
A reabilitação de pacientes que sofreram TCE é complexa pelo quadro de déficit neurológico geralmente presentes nestes indivíduos. Mesmo que persistam baixos níveis cognitivos, a fisioterapia aquática não é contra-indicada, a não ser que represente risco de vida ou de acidente ao paciente ou ao terapeuta ${ }^{16}$.

O objetivo deste estudo é avaliar os efeitos sensóriomotores no paciente com seqüela de TCE através do tratamento precoce em fisioterapia aquática, enquanto o paciente ainda se encontre em estado de coma vigil.

O estudo consiste em um relato de caso, paciente APC, 40 anos, sexo feminino, com seqüelas neurológicas pós TCE, em estado de coma vigil, nível cognitivo II (escala Rancho Los Amigos) ${ }^{8}$, após um ano de internação. Estabilizado clinicamente, na avaliação fisioterapêutica, apresenta espasticidade global nível III (escala Ashworth) ${ }^{17}$, movimentação não seletiva de membros superiores e inferiores com predominância do hemicorpo direito, o controle cervical e de tronco na avaliação estática e dinâmica encontram-se precários e apresentando hiperreflexia e movimentação involuntária do membro inferior esquerdo. Apresenta abertura ocular espontânea e não faz acompanhamento visual dirigido.

Após avaliação multidisciplinar foi iniciada a fisioterapia aquática, no qual seu protocolo baseou-se em estimulação vestibular. O mesmo consistia em imersão do ouvido, adequação tônica, relaxamentos globais (deslocamentos e flutuações), estimulação das etapas posturais (mobilizações passivas tridimensionais), estimulação cognitiva e adequação ambiental.

O tratamento aquático teve como base o método Halliwick, Bad Ragaz e Watsu, específicos da fisioterapia aquática, porém adaptados de métodos terrestres bem como Bobath, Kabat, RPG e Shiatsu, com finalidade terapêutica ${ }^{15}$.

O estudo foi realizado em 18 meses, três sessões semanais com duração de 45 minutos. Foi feita uma avaliação inicial, utilizando as escalas Rancho Los Amigos e Ashworth para mensuração estatística, rea- valiado mensalmente, mantendo o mesmo protocolo de tratamento e não realizando terapia terrestre.

No contexto sensorial, observou-se no presente estudo, a melhora da conscientização espacial e cognitiva, através da análise dos escores inicial (II) e final (IV) da escala Rancho Los Amigos, encontrando-se em elevação.

Analisando as aquisições motoras, podemos constatar melhora do controle cervical, ajuste e adequação tônica, melhora do padrão postural e redução do reflexo do membro inferior esquerdo.

Em se tratando da espasticidade não houve melhora significativa perante a escala de Ashworth, no entanto, não se desenvolveram instalações de encurtamentos e deformidades, representando um foco de grande importância à manutenção da integridade das amplitudes de movimentos articulares.

Levando em consideração que o tratamento é precoce, a manutenção das amplitudes de movimentos é de suma importância, pois irão potencializar os resultados motores voluntários posteriores.

Concluímos que o paciente em coma vigil beneficiou-se com a intervenção da fisioterapia aquática, pois desenvolveu ganhos motores e sensoriais, além de estar músculo-esqueleticamente sem limitações para realizar posterior movimentação voluntária. A mesma poderá ser melhor orientada e desenvolvida quando o paciente se encontrar fora do estado de coma vigil. Sabe-se que, desde já, possui toda a possibilidade motora (articular e flexibilidade muscular) para estas aquisições, além de estarem sendo desenvolvidas.

Por se tratar de um estudo de caso não podemos nos certificar que todo o paciente em coma vigil obterá resultados positivos na imersão precoce como o paciente em estudo. Dessa forma sugerimos um estudo analítico e experimental com um grupo de pacientes, para que os mesmos possam ser avaliados e concluir se há melhora em um percentual significativo para embasamento científico. 


\section{REFERÊNCIAS BIBLIOGRÁFICAS}

1. Michaud, L.; Duhaime, A.; Batshaw, M. Traumatic Rain Injury in Children. Pediatric Clinic of North America, v. 40, n.3, p. 553-565, 1993.

2. Tecklin, J.S. Pediatric Physical Therapy, $3^{a}$ ed., Lippincot, 1998

3. Guerra, S. et al Traumatismo Crânio Encefálico em Pediatria. Jornal de pediatria, Rio de Janeiro, n.75, p.279-293, 1999.

4. Mizumoto, N.; Tango, H. K.; Pagnocca, M. L.. Efeitos da hipertensão arterial induzida sobre a complacência e pressão de perfusão encefálica em hipertensão intracraniana experimental: comparação entre lesão encefálica criogenia e balão subdural.Rev. bras. anestesiol., 55(3):289-307, maio-jun. 2005.

5. Stokes, M. Neurologia para Fisioterapeutas, ed Premier, São Paulo, 2000.

6. Rosenthal, M., Whyte J. Reabilitação do Paciente com Traumatismo Craniano. In Delisa, J. Medicina de Reabilitação: Princípios e Práticas, ed. Manole, v. 2, cap 30, pp. 677-708, 1992.

7. Jennett, B.; Snoek, J.; Bond, M.R.; et al. Disability After Severe Head Injury: Observations on the Use of the Glasgow Outcome Scale, 44:285-293, J. Neurol. Neurosurg Phychiat, Philadelphia, 1981.

8. Chris Hagen, Ph.D., Danese Malkmus, M.A., Patricia Durham, M.A. Levels of Cognitive Functioning, Communication Disorders Service, Rancho Los Amigos Hospital, California, 2002
9. Hall, D.M. et al. Rehabilitation of Head Injured Children. Archives of Diseases in Childhood, n.65, p. 553-556, 1990.

10. Ribas, G; Gherpelli, J.L.; Manresa, L. traumatismo Crânio Encefálico. In: Diament A. e Cypel, S. Neurologia Infantil, ed. Ateneu, $3^{a}$ edição, cap. 84, pp 1198-1214, 1996.

11. Di Scala, C. et al Functional Outcome in Children with Traumatic Brain Injury: Agreement between Clinical Judgment and the Functional Independence Measure. Am J Phys Med Rehabil, n.71, p.145-148, 1992.

12. Oliveira, C.E.N.; Salina, M.E.; Anunciatto, N. Fatores Ambientais que Influenciam a Plasticidade do SNC. Acta Fisiatrica.n.8, p.6-13, 2001

13. Gimenes, R.O; et al. Análise crítica de ensaios clínicos aleatórios sobre fisioterapia aquática para pacientes neurológicos, revista neurociências, vol $13 n^{\circ} 1$ São Paulo, 2005

14. Becker,L.R. Enthusiastic physical therapists jump feet first into aquatic research. Advance for Physical Therapists, 4(22):10, Boston, 1993

15. Ruoti, Richard, et al. Reabilitação Aquática, ed. Manole, São Paulo, 2000

16. Campion, M. Hidroterapia: Princípios e Prática, ed. Manole, São Paulo, 1999.

17. Souza, A.M., Ferrareto, I. Paralisia Cerebral: Aspectos Práticos, ed. Memon, $2^{a}$ edição, São Paulo, 2001. 